\title{
Discussion on the Phenomenon of Irrational Clinical Drug Use and Improvement Measures
}

\author{
Liu Jing-wen \\ Tianjin Second Hospital Tianjin, Hebei District, Tianjin 300141 \\ https://orcid.org/0000-0002-7575-6721
}

Received: 16 December 2019 Accepted: 25 December 2019 Published: 31 March 2020

Cite this Article Liu Jing-wen. Discussion on the Phenomenon of Irrational Clinical Drug Use and Improvement Measures [J].Medical Research,2020.2(1): 5-9, http://dx.doi.org/10.6913/MRHK.20200 3_2(1).0002

Copyright (C) 2020 Creative Publishing Co., Limited. All rights reserved. Email: kycbshk@gmail.com.

\begin{abstract}
Objective To discuss the irrational drug use in clinic and its improvement measures.

Methods To select 132 prescriptions of Western medicine from the pharmacy department of our hospital as the research object, prescribed from July 2017 to September 2018. According to the prescription time, the safety and irrational use of drugs were observed according to the principle of clinical rational use of drugs. They were divided into experimental group and control group, in which the control group did not include the improvement measures, the experimental group included the improvement measures and each group had sixty-six prescriptions.
\end{abstract}

Results The incidence of repeated drug use, improper drug use combination, improper use of antibiotics, the abuse of antibiotics and other irrational drug uses in the experiment was lower than that in the control group and there was significant difference between the two groups $(\mathrm{P}<$ 0.05).

Conclusion In the clinical drugs administration of Western medicine, it is necessary to strengthen the observation of irrational drug use and give timely improvement measures to ensure the safety of clinical drugs.

Key words Irrational drug use; Improvement measures; Clinic

Western medicine is a commonly used drug in clinical treatment. With the continuous development of medical and health undertakings, the variety of drugs is increasing. From the point of view of clinical treatment, western medicine has the characteristics of short treatment cycle and good effect, so it is widely used [1]. Because there are many kinds of Western medicine, some components of Western medicine have some similarities, while some clinical pharmacists not only are not familiar with drugs, but also don't know about the DOs and DON'TS (Taboos) of drug use, so there will be some irrational drug use in clinic. This phenomenon not only increases the drug resistance of patients, affects the treatment effect of patients, but also reduces the clinical treatment effect and even leads to the emergence of medical accidents. Therefore, clinical pharmacists and physicians should master the rational use of drugs, DOs and DON'TS (Taboos) for application and related pharmacological knowledge, so as to ensure the 
rationality of clinical drug use. In this study, our hospital's pharmacy department selected 132 western medicine prescriptions as the research object from July 2017 to September 2018. According to the prescription content, this study is to discuss the safety of rational clinical use of Western medicine and implement the improvement measures according to the situation. The specific situation was as follows.

\section{DATA AND METHODS}

\subsection{General data}

At present, there were 132 prescriptions of Western medicine selected from pharmacy department of our hospital as research objects. The prescription time is July 2017-September 2018. According to the prescription time and according to the principle of clinical rational drug use, observe the safety and irrational drug use. They are divided into experimental group and control group, in which the control group didn't include the improvement measures, the experimental group included the improvement measures and there are 66 pieces of prescriptions per each group. In the experimental group, there were 6 pediatric prescriptions, 10 gynecological and obstetric prescriptions, 8 cardiac surgery prescriptions, 7 thoracic surgery prescriptions, 14 urological surgery prescriptions, 13 general surgery prescriptions and 8 neurosurgery prescriptions. In the control group, there were 18 pediatric prescriptions, 12 gynecological and obstetric prescriptions, 11 cardiac surgery prescriptions, 8 thoracic surgery prescriptions, 12 urological surgery prescriptions, 17 general surgery prescriptions and 6 neurosurgery prescriptions. There was no statistical significance in general data between the two groups $(\mathrm{P}>0.05)$.

\subsection{Methods}

Relevant regulations make irrational and scientific records for 132 prescriptions of Western medicine in terms of types, routes of use, usage and dosage, combined use of drugs and individual use of drugs, summarizing the causes of adverse clinical rational use of drugs. Additionally, they formulate the corresponding measures according to the causes. The irrational drug use mainly includes the following aspects.

\subsubsection{Non-standard use of antibiotics in perioperative period}

The main purpose of perioperative medication is to prevent the infection of surgical site, including the infection of organs and incisions involved in the operation, but not the infection that has no direct relationship with the operation. However, the prevention of postoperative infection often occurs in the actual medication.

\subsection{The irrational use of dose and the use of contraindication drugs}

In clinic, there are often random changes in drug dosage, or cases of missed or forgotten drugs taking. Rational drug dosage is formulated through effective clinical experiments and rational drug use theory. This kind of random drug use behavior directly affects the therapeutic effect [4], and even causes adverse reactions in patients. The irrational use is mainly due to the excessive dosage, which may lead to poisoning, especially in the use of drugs for children and the elderly, the risk is very high. At the same time, the reduction of drug dosage will not only not achieve the therapeutic effect, but also delay the patient's condition, and even produce drug resistance. Drug contraindications generally occur in the case of poor guidance from clinicians and pharmacists. Because patients lack effective guidance, they are not aware of their drug contraindications. 
In the treatment of diseases, many patients have a mentality that the effect of combined use of multiple drugs is better than that of single drug. But in the actual clinical treatment, if blindly combined with drugs, it will not only not increase the therapeutic effect, but also cause other hazards [5]. First of all: in many drugs, the composition of the drug is the same, but the trade mark of the drug is different, so it will lead to repeated drug use, which is the cumulative dose of the drug. Secondly, in many drug applications, there is no reasonable combination of drugs according to the instructions, resulting in incompatibility taboos, drug failure, and even toxic reactions [6]. Finally, many drugs are not used rationally, especially the use of antibiotics, which is prone to drug resistance and is not conducive to clinical treatment. Therefore, rational use of clinical drugs is needed. In view of the above situation, our hospital has taken a series of improvement measures mainly for the irrational drug use behavior improvement, the results are as follows.

\subsection{Observation Indexes}

The incidence of irrational drug use in prescriptions was compared, including repeated administration, improper combination of drugs, improper use and dosage, abuse of antibiotics and other irrational drug use.

\subsection{Statistical Methods}

Relevant data from the survey were input into SPSS17.0 statistical software package for processing. The incidence of irrational drug use was described by $n(\%)$. The difference between groups was tested by $\mathrm{x}^{2}$. When the difference was $\mathrm{P}<0.05$, it showed that there was clinical comparable significance.

\section{RESULTS}

The incidence of repeated drug use, improper drug use combination, improper use of antibiotics, the abuse of antibiotics and other irrational drugs in the experiment was lower than that in the control group and there was significant difference between the two groups $(\mathrm{P}<0.05)$, as shown in Table 1.

Table 1 Comparison of the Incidence of Irrational Drug Use in Two Groups

\begin{tabular}{|c|c|c|c|c|c|c|c|}
\hline Group & $\begin{array}{l}\text { Number } \\
\text { of cases }\end{array}$ & $\begin{array}{c}\text { Improper } \\
\text { drug use } \\
\text { combination }\end{array}$ & $\begin{array}{c}\text { Improper } \\
\text { methods and } \\
\text { does }\end{array}$ & $\begin{array}{c}\text { Improper } \\
\text { use of } \\
\text { antibiotics }\end{array}$ & $\begin{array}{l}\text { Repeated } \\
\text { drug use }\end{array}$ & Others & $\begin{array}{c}\text { Incidence of } \\
\text { irrational } \\
\text { drug use }\end{array}$ \\
\hline $\begin{array}{l}\text { Experimen } \\
\text { tal group }\end{array}$ & 66 & 1 & 1 & 1 & 0 & 0 & $3(4.54)$ \\
\hline $\begin{array}{l}\text { Control } \\
\text { group } \\
\mathrm{X}^{2} \\
\mathrm{P}\end{array}$ & 66 & 4 & 3 & 6 & 3 & 2 & $\begin{array}{c}18 \\
(27.27) \\
12.7413 \\
0.0003\end{array}$ \\
\hline
\end{tabular}

\section{DISCUSSION}

In recent years, our country attaches great importance to clinical rational drug use and requires clinical pharmacists and clinicians to guide clinical drug use in accordance with the Guiding Principles of Antibiotics, including rational drug use in surgery, basic drug use principles and drug compatibility taboos, etc. [7]. This paper summarizes the common irrational drug use phenomena, and takes rational measures to improve it. The specific measures are as follows. 


\subsection{Establishment of a Complete Drug Administration Organization}

To formulate basic drug use catalogue and the principle of drug selection to evaluate the safety of the new products in the hospital. To give regular lectures on conventional drug use knowledge and offer classes or seminars. To enhance the correct diagnosis of common diseases by medical staff,meanwhile it is necessary to adopt the opinions of clinical licensed pharmacists in order to ensure the rationality of drug use. To make the reward and punishment policies for clinical use of drugs, reward medical personnel for rational use of drugs and promote their experience.

\subsection{Establishment of Scientific Pharmaceutical Management Model}

In the treatment of patients, it is necessary to take the patient as the center instead of the original disease-centered treatment model. Establish a special drug quality management department in the hospital, which is mainly responsible for the comment of prescriptions. Give praise and publicity to representative prescriptions that use reasonable prescriptions. At the same time, focus on the use of unreasonable prescriptions, and explain to medical staff how to avoid the same problem. Through the above means to establish a sound pharmaceutical management model to ensure the rationality of drug use.

\subsection{A Guarantee of Good Medical Ethics and Customs}

In many hospitals, there are drug marketers. In order to increase drug sales, they will carry out inappropriate marketing behavior to doctors. Some doctors randomly increase the types of drugs in front of interests, resulting in the generation of unreasonable drugs. Therefore, it is very important to maintain good medical ethics in the environment. We should uphold the concept of serving the people wholeheartedly and maintain high-quality medical behavior.

After analyzing the causes of clinical irrational drug use and taking appropriate improvement measures, it was found that the repeated drug use, improper drug use combination, improper use of antibiotics, the abuse of antibiotics and other irrational drugs in the experimental group was lower than that in the control group and there was significant difference between the two groups $(\mathrm{P}<0.05)$. To sum up, in the clinical drug administration of Western medicine, it is necessary to strengthen the observation of its irrational use of drugs and give timely improvement measures to ensure the safety of clinical drug use.

\section{REFERENCES}

[1] Gao Jianzhen, Li Jiwen. Exploration of the safety and countermeasures of clinical rational drug use for western medicine [J]. Health People (Academic Edition), 2014, 8 (19): 315.

[2]Ozbek Aberiba. Analysis on the safety and countermeasures of clinical rational drug use of western medicine[J]. World Latest Medicine Information,2016,16(40):85-86.

[3]Zhang Yuxiu.Safety and Countermeasures of rational clinical use of Western medicine[J].Chinese Medical Guidelines, 2016,14(16): 49.

[4] Shen Wei. Analysis of the safety and management countermeasures of clinical rational drug use in western medicine[J]. Continuing Medical Education, 2016, 8(10): 146-147.

[5] Tang Li. Safety and countermeasures of clinical rational drug use in western medicine[J]. China continuing medical education, 2015, 7(8): 235.

[6]Yu Qin, Han Lili, Zhang Yan'e. To explore the safety of rational clinical use of Western medicine and the corresponding measures [J]. Chinese Medical Guidelines, 2017, 15 (7): 119-120.

[7] Xiao Ailing. Exploration of the safety and countermeasures of clinical rational drug use for western 

medicine[J]. Chinese Health Standards Management, 2017, 8(9): 56-57.

[8]An Xuemei. Analysis of the safety and countermeasures of clinical rational drug use in western medicine[J].China Practical Medicine,2017,12(22):93-94. 